\title{
How Companies are Applying AI to the Business Strategy Formulation
}

\author{
Prof Dr M S S EI Namaki \\ Dean, Victoria University, School of Management, Switzerland. \\ Dean (Retired) Maastricht School of Management, MSM, The Netherlands.
}

\begin{abstract}
I The Problem
Computing equipment capable of what one may term partial and quasi-intelligent behaviour, commonly referred to as Artificial Intelligence (AI), is assuming a key role in business. The probability is high that this Artificial Intelligence (AI) will lead to a fundamental change in the process of business strategy formulation as much as the very contents of this strategic behaviour. Product and market strategies and the resultant competitive behaviour will, more likely than not, be the outcome of those artificial intelligence processes and reiterations. A start is made and one can observe substantial progress in this direction.
\end{abstract}

Who has done it and is there a conceptual framework behind this strategic behaviour? This will be the focus of this article

The article starts with a brief definition of artificial intelligence and a basic framework of the concept. Seven case studies follow supporting the hypothesis that AI is penetrating the business strategy arena and leading to a fundamental change in the concept as much as the application. Those cases were drawn from different industries, and countries. A conceptual framework is accordingly derived and positioning of those case companies within this conceptual framework is done.

The article is based on contemporary frameworks of AI and the cases are drawn from contemporary analysis of strategic behaviour. The conceptual model could provide an instrument for business AI application.

Keywords: Artificial Intelligence, AI, Business Strategy, Policy, Technology

\section{What is Artificial Intelligence AI?}

Merriam-Webster defines artificial intelligence as "A branch of computer science dealing with the simulation of intelligent behaviour....." and "the capability of a machine to imitate intelligent human behaviour." (Forbes, Feb 14, 2018,). Put differently, AI is the simulation of human intelligence processes by machines, especially computer systems. These processes include learning (the acquisition of information and rules for the use of this information), reasoning (using rules to reach approximate or definite conclusions) and self-correction (self-initiated adjustment or mending of errors and malfunctions). AI relates to many sciences from computing, mathematics and data to psychology, philosophy and linguistics.

The Artificial Intelligence concept lends itself to segmentation. There is segmentation by time, by the complexity and by genericness. Let us confine ourselves to the time segmentation. 


\section{- Present day Artificial Intelligence :}

These present day intelligent systems are able to handle massive volumes of data but lack the analytical and independent self-awareness element that will be key to building future intelligence. They are either reactive or limited memory.

- Reactive. These are equipment that analyzes possible moves, their own and their opponent's, and choose the most strategic move. They do not have the ability either to form memories or to use past experiences in order to guide current decisions. The computer's perception of the world is direct and it acts according to what it sees.

- Limited memory (corrective). These equipment use past experience in order to influence future decisions. They can look into the past but past information is only transient and are not saved as part of a library or a learning experience.

(The Conversation, November 14, 2016)

\section{- Future Event Artificial Intelligence:}

Systems within this AI segment do not only form representations about its own world but also about other agents or entities in the world. They do not only understand consciousness but have it.

- Theory of mind. This is a psychology term. It refers to the understanding that others have beliefs, desires and intentions that impact upon the decisions that they make. Also, those others have beliefs, desires, intentions, and perspectives that are different from one's own. This kind of AI does not yet exist.

- Self-awareness. In this category, AI systems have a sense of self and consciousness. Machines with self-awareness understand their current state and can use the information to infer what others are feeling. Conscious beings are aware of them, know about their internal states, and are able to predict feelings of others. This type of AI does not yet exist. (The Conversation, November 14, 2016)

Algorithms are central to the concept of AI. An algorithm is a set of unambiguous instructions that mechanical computing equipment can execute. ${ }^{[b]}$ A complex algorithm is often built on top of other, simpler, algorithms (Russell \&Norvig 2003),

AI conceptual framework is evolving at exceptional speed. It seems to project the core of a fifth industrial revolution.

\section{Did Artificial Intelligence Influence Business Strategy Formulation?}

It is the author's contention that recent strategic moves by several corporations Worldwide have been in response to AI events or outcomes. The following are some of those cases where a decision and a strategy emerged as a result of an AI development. A pattern could be distilled. This pattern will be demonstrated in a conceptual model.

\section{Case One: \\ IBM, E-tailing and GWYN}

Online shopping reveals a preference, spending habits and channel choice data of individual consumers. Feeding these digital bits of information into an AI construct helps bring curated shopping journeys to mass audiences. IBM Watson provides an illustration. The software, known as GWYN, provides a range of order management and customer engagement capabilities to e-commerce retailers through information provided by consumers about a gift recipient. The software tailors gift recommendations by comparing specifics provided to gifts purchased for similar recipients. GWYN attempts to replicate the role of a concierge at a store through a personal and detailed conversation with users. (Techno emergence Oct 28, 2018). . It provides seamless customer service experience based on consumers purchase history and known preferences. 


\section{Case Two:}

\section{Tangli and Huawei, smart cities and Digital Platform}

Artificial Intelligence is helping address urban challenges by enhancing the effectiveness of city and urban management. Huawei developed a smart city digital platform utilizing artificial intelligence (AI) and the Internet of Things (IoT) capabilities in order to manage public safety, environmental protection, transportation, government, education, and agriculture. Huawei's digital platform connects what it calls the brain, the central nervous system, and a peripheral nervous system, made up of sensors across a city. The platform is compatible with different city sensors, creating a city digital twin, and supporting diverse city applications. TANGLI, a Chinese data science operator, has also developed a similar smart city formula. It is similar to Huawei in terms of the resort to sensors to collect data that is later subjected to AI analysis with an outcome put to use in urban city management. And so on.

\section{Case Three:}

Beyer, Pharma and PVAI

Pharma industry is set to benefit from AI in a wide number of areas from drug discovery and testing to drug manufacturing, diagnostic assistance, medical treatment process and application monitoring. Bayer, the German pharma maker, is enhancing its patient safety data monitoring by resorting to AI in an attempt at early tracing of drug-related side effects. Pharmacovigilance Artificial Intelligence (PVAI) or the PVAI solution, which extracts adverse event data from source documents in an automated fashion, has taken part in and won, a number of competitive proof-of-concept trials run by several large pharmaceutical companies. The ability to apply advanced AI technologies, with significant process and domain expertise, will usher in a whole new era of pharmacovigilance. It will serve as a single machine learning system that can be utilized across the healthcare industry by life sciences companies, healthcare providers, and regulators. (Pharmaphorum, Nov 2, 2018).

\section{Case Four:}

NATO, Defense and AICA

AI is changing the very nature of the defence industry with hardware rapidly taking a second place to software. NATO research points to intelligent, partly autonomous agents becoming primary cyber fighters on the future battlefield. Those intelligent software agents would perform, then, active, largely autonomous cyber defence actions within military computerized networks. A situation is envisaged where enemy software cyber agents - malwarewould infiltrate friendly networks and attack friendly computerized command, control, communications, intelligence, surveillance, and reconnaissance weapon systems. To fight this hostile "software", NATO is developing artificial cyber hunters-intelligent, autonomous, mobile agents specialized in active cyber defence. That Autonomous Intelligent Cyber Defense Agents (AICA) is more likely than not to be the product of AI research. (ARL: March 2018) (Kott, March 2018).

Case Five:

Fujitsu, Product Development and FTCP

Artificial intelligence technologies enhance the process of product development. New technologies based on machine learning accurately predict future product design data needs and replace past human input. Fujitsu resorted to those AI instruments in order to provide computerized support to various product development tasks. A "Flexible Technical Computing Platform (FTCP)", was created in order to facilitate efficient collection of product development data, and, at the same time, for managing and leveraging learning models extracted from such data. (Application of artificial intelligence technology in product design. Nov 10, 2018): Product development process has been transformed from the traditional sequential process into a dynamic concurrent, simultaneous and parallel process.

\section{Case Six:}

Shape Security, Cybersecurity and Blackfish

http://dx.doi.org/10.19085/journal.sijbpg050801 
Security of financial transactions is becoming a key issue on the light of Fintech and the radical shift to online transactions. AI is playing a key role in enhancing this cybersecurity as well as fraud detection. Companies providing AI-based cybersecurity solutions for major financial institutions are increasing in number and reach. Shape Security is a US cybersecurity firm that resorts to machine learning in order to curb credit application fraud and credential theft. The company's machine learning models expose application data to software that effectively distinguishes between bona fide consumers and doubtful cases. The company's "Blackfish" network also uses AIenabled bots to detect compromised login credentials, alerting both customers and companies to security breaches.

\section{Case Seven:}

Qopius, logistics and Vision Based Inventory

AI is supporting the digitalization of logistics. Computer vision inventory management software is being introduced in the retail industry. Key supply chain operators are embarking upon substantial digital transformation of the industry. French startup Qopius is developing computer vision-based AI to measure shelf performance, track products, and improve retail store operations. Using deep learning and fine-grained image recognition, Qopius is able to extract attributes of items such as brand, labels, logos, price tags, as well as shelf condition ( out of stock, the share of shelf, and on-shelf availability). Similar use of computer vision offers the potential for real-time inventory management support as well. AI will accelerate the path towards a proactive, predictive, automated, and personalized future for logistics. (DHL, 2018)

\section{A Conceptual Framework}

It is the author's contention that cases analyzed above carry the traces of a conceptual framework relating artificial intelligence to the strategic behaviour of those firms. This conceptual framework identifies two AI related variables as determinants of situational strategic behaviour. The first variable is functions to be performed within an environment and the second variable is the very markets where function delivering instruments are exchanged. Both variables can be projected within a matrix where the $\mathrm{x}$-axis represents function delivery products (or instruments) and the $y$-axis represents function-related markets.

Figure

Patterns of AI Compatible Strategic Behavior

Function Fulfilling Technologies/ Instruments

Existing

Future

Function-Related Markets

\begin{tabular}{|c|c|c|c|c|}
\hline Existing & I. & $\begin{array}{l}\text { Existing technologies for } \\
\text { existing markets }\end{array}$ & II. & $\begin{array}{l}\text { AI Developed Functions } \\
\text { Fulfilling Substitute } \\
\text { (Huawei Urban Mgt.) }\end{array}$ \\
\hline Future & & $\begin{array}{l}\text { Novel AI Developed } \\
\text { Markets for Existing } \\
\text { Products } \\
\text { er Pharmaceutical Testing) }\end{array}$ & $I V$. & $\begin{array}{l}\text { AI Developed } \\
\text { Technologies for New } \\
\text { Markets (NATO AICA) }\end{array}$ \\
\hline
\end{tabular}

http://dx.doi.org/10.19085/journal.sijbpg050801 
The matrix identifies four patterns of AI compatible strategic behaviour:

- Static behaviour. Status quo. Existing technologies for existing markets. A pre-AI phase.

- AI technologies providing function fulfilling substitutes to existing products or instruments within the same market. Strategic behaviour aims at endorsing and leveraging AI added value.

- AI developed novel functions and function fulfilling technologies for novel markets. Strategic behaviour here aims at the advent of new industries and markets.

- Novel AI derived markets for existing products or technologies. Strategic behaviour here addresses the needs of the new markets and seeks compliance with the requirements of that market.

Four distinctive states each with a measure of AI influence.

\section{Implications for the Strategic Thinking Concept}

Conceiving AI dynamics along the lines of the suggested matrix will, very likely, lead to a significant shift in approaches to business strategic thinking as much as the very content of the strategies. Several hypotheses could emerge from this analysis and the following are only the author's immediate formulations:

- Products and services are functions fulfilling instruments and not market derivative.AI derived algorithms will lead to optimum function fulfilling technologies, and products, replacing present day R and D outcomes.

- Time will assume a different scale. The fast evolution of AI will lead to a different view of time and the measurement of this time within a strategic framework. The long time term of yesteryear will, more likely than not, become a short time term. The parameter is not minutes and hours but events!

- Capitalism could exist without capital. The development of AI technologies may not require the massive assembly of capital observed during the third or the fourth industrial revolution.

- Industries will change the structure and assume different structural parameters. AI will lead to as much vertical as horizontal shifts within industries.

Future research could test these hypotheses.

\section{Summary and Conclusions}

$\mathrm{AI}$ is a strategic industry with a potentially far-reaching impact on business and government. There are those who think that a fifth industrial revolution seems to be on the horizon with artificial intelligence as the driver.

AI corporate reach is likely to cover every functional and strategic dimension of business. Issues as product development, market development, supply chain, consumer behaviour, information systems and many others will all be closely related to AI instruments and outcomes. Data science induced algorithms will very likely hold the answer to longer-term strategies and actions within many industries from manufacturing and finance to education, health care, media and retailing.

The article reviews seven cases and derives a conceptual framework that would allow a measure of operational projection of AI strategy interface. Conceiving AI dynamics along the lines of a suggested matrix will lead to a significant shift in approaches to business strategic thinking as much as the very content of the concept and the strategies. And the article draws broad conclusions as to the impact of AI on the strategic thinking process.

\section{References}

[1]. Understanding the four types of AI, from reactive robots to self-aware beings, The Conversation. November 14, $2016^{\prime \prime}$

[2]. Alexander Kott et al "Initial Reference Architecture of an Intelligent Autonomous Agent for Cyber Defense", US Army Research Laboratory, March 2018.

[3]. Bayer applies artificial intelligence to its pharmacovigilance systems, harmaphorum, November 2, 2018.

[4]. Artificial Intelligence in Retail, Technoemergence, Oct 28,2018. 
[5]. Artificial Intelligence in logistics, A collaborative report by DHL and IBM on implications and use cases for the logistics industry, 2018

[6]. Preparing for the future of Artificial Intelligence, National Science and Technology Council Artificial Intelligence, Automation, and the Economy, 2016 Executive office of the President, International Journal of Engineering and Innovative Technology (IJEIT), Volume 4, Issue 10, April 2015

[7]. How Artificial Intelligence is Transforming Product and Machine Design, G2 Crowd January 20, 2017.

[8]. Bernard Marr, The Key Definitions Of Artificial Intelligence (AI) That Explain Its Importance, Forbes, Feb 14, 2018.

[9]. Transforming Product Development and Design, https://eteam.io/blog/ai-and-product-dvelopment-design/

[10]. Application of artificial intelligence technology in product design. Available from:

https://www.researchgate.net/publication/318860126_Application_of_artificial_intelligence_technology_in_produc t_design [accessed Oct 27 2018].

Irving Wladawsky-Berger,The Impact of Artificial Intelligence on R\&D and Innovation WSJ, June 15, 2018

[11]. McKinsey Global Institute, "The Promise and Challenge of the Age of Artificial Intelligence", October 2018.

[12]. AREND HINTZE, Understanding the Four Types of Artificial Intelligence, GT, NOVEMBER 14, 2016 